\title{
Radiation detection: novel approaches and readout capabilities exploiting latchup topology via bipolar, MOSFET and MESFET transistors
}

\author{
Alessandro Gabrielli ${ }^{1}$ \\ INFN \& Physics Department, University of Bologna \\ Viale Berti Pichat 6/2 40127 Bologna, Italy \\ E-mail: alessandro.gabrielli@bo.infn.it

\section{Enrico Giulio Villani} \\ STFC Rutherford Appleton Laboratory Particle Physics Dept., \\ Chilton, Didcot U.K. \\ E-mail: giulio.villani@stfc.ac.uk
}

\begin{abstract}
The stimulated ignition of latchup effects caused by external radiation has so far proven to be a hidden hazard. Here this effect is described as a novel approach to detect particles by means of a solid-state device susceptible to latchup effects. In addition, the device can also be used as a circuit for reading sensors devices, leaving the sensing capability to external sensors. The paper describes the state-of-the-art of the proposal and its development over the latest years and then the present and future studies are shown. The study begins using traditional bipolar transistors since the latchup effect is originated as a parasitic circuit composed of such devices. Then, an equivalent circuit built up of MOS transistors is exploited, resulting an even more promising and challenging configuration than that obtained via bipolar transistors. For this, an elementary cell composed of two transistors connected in a thyristor structure is presented. Eventually, also a circuit implementing a MESFET commercial component confirms the basic principle. As the MOS transistors are widely used at present in microelectronics devices and sensors, a latchupbased cell is proposed as a novel structure for future applications in particle detection, amplification of signal sensors and radiation monitoring.
\end{abstract}

9th International Conference on Large Scale Applications and Radiation Hardness of Semiconductor Detectors-Rd09

Florence, Italy

30 September - 2 October 2009

\footnotetext{
${ }^{1}$ Speaker: Alessandro Gabrielli
} 


\section{Introduction}

This paper presents a work that started just few years ago, when the authors were investigating redundant logic circuits against Single Event Effects (SEE) [1] and studying new structures to reduce the in-pixel power consumption, respectively. In particular, SEE originate when an over-threshold charge is deposited in specific points, called sensible nodes, of microelectronics devices. Hence, while studying and investigating on these effects we started exploiting one of the most dangerous of the SEEs: the latchup effect [2]. This effect could be exploited as a powerful means of achieving the precise detection and positioning of a broad range of ionizing particles or, for example, the proposed device can only be used as a readout circuit for amplification and latching of a variety of signals provided by sensors. In fact, the circuit takes the function of the data acquisition chain that is today designed within each pixel of pixel detectors. These are widely used, for example, in the experiments [3, 4, 5] of the Large Hadron Collider. Although the principle was already proved in the past [6, 7], a novel prototype has been designed, constructed and tested and some new results are presented below.

Future applications in high-energy physics and in radiation monitoring seem to be the most suitable for this type of device. In addition, for its high simplicity and, consequently, for its very low power consumption, it is also easily adaptable to a wide range of radiation monitors, from portable devices to huge pixel detectors.

\section{The novel prototype}

Figure 1 shows two MOS transistors instead of the bipolar devices that create the wellknown latchup circuit (i.e a thyrstor). Figure 2 shows how the circuit has been implemented via commercial MOS components. In more detail, by connecting the MOS transistors extracted from CMOS inverters after having disconnected the power pin of the N-MOS and the ground pin of the P-MOS, the two individual transistors are available to be connected as in Fig. 1. In this way we tried to exploit submicron MOS transistors without fabricating an integrated version of the cell, which is to be done in the next future in any case.

Thus, we have constructed a board to really test the latchup circuit in laboratory. Figure 3 shows the board; it has been provided with many jumpers and variable resistors to easily configure and bias in several ways the circuit shown in Fig. 1.

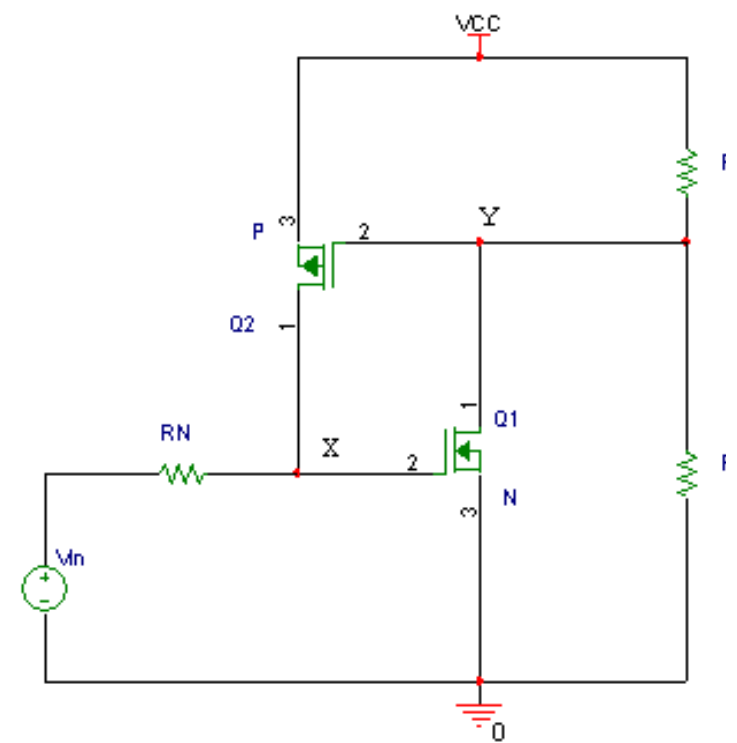

Figure 1: Latchup circuit 
Figure 4 shows an oscilloscope plot of the cell under test, realized via commercial MOS transistors extracted from commercial inverters as shown in Fig. 2 and implemented via the board shown in Fig. 3. By following the top graph from left to right, it is evident that initially the output signal is at high (supply) voltage. This indicates that the entire thyristor is off, waiting for a latchup ignition. Then, an over-threshold spike is provided with the NMOS gate (bottom graph) and, as a consequence, the output voltage goes down to reach its standing value. Here the situation stabilizes and the circuit locks into a standing condition. Successively, a reset pulse not shown in the figure forces the circuit into the initial turned off condition. This pulse is provided through an additional switch (a third MOS transistor) that short circuits the $\mathrm{N}$ MOS's gate to ground. After having proved that the circuit effectively ignites depending on the input spike, we have measured the spike height, which is of the order of $10 \mathrm{mV}$, and the input impedance of the circuit, which is of the order of $100 \Omega$. As the pulse width is about $100 \mathrm{~ns}$, the injected charge in the transistor's gate is of the order of $10 \mathrm{pC}$ $(10 \mathrm{mV} / 100 \Omega \times 100 \mathrm{~ns}=10 \mathrm{pC})$. This is a rough estimation fully compatible with what was obtained in [6, 7].

Eventually, we measured the noise figure of the circuit in terms of spread in the ignition voltage. Hence, we have swept accurately the spike height, while measuring the ignition-to-non-ignition ratio over 200 cycles at a time. More precisely, we have repeated the tests by increasing the height of the spike starting from an initial value that did not ignite the circuit at any time. Then, we have stopped increasing the pulse height when we reached a situation in which the circuit always ignited as soon as the pulse was provided and

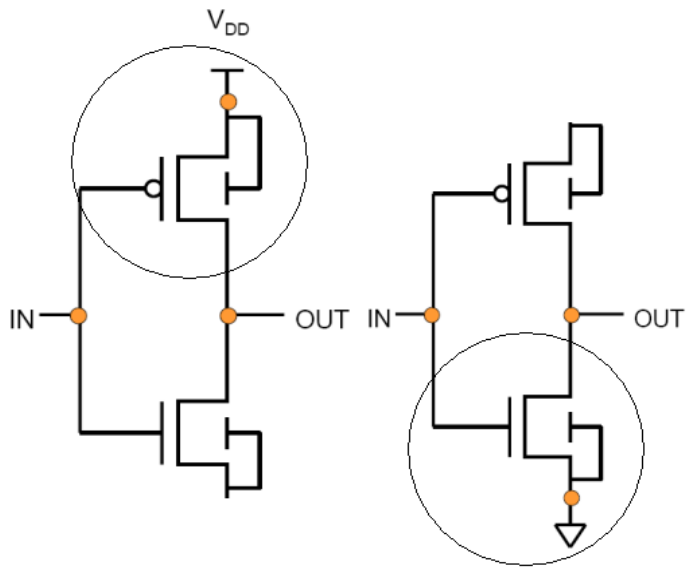

Figure 2: Transistors extracted from commercial inverters

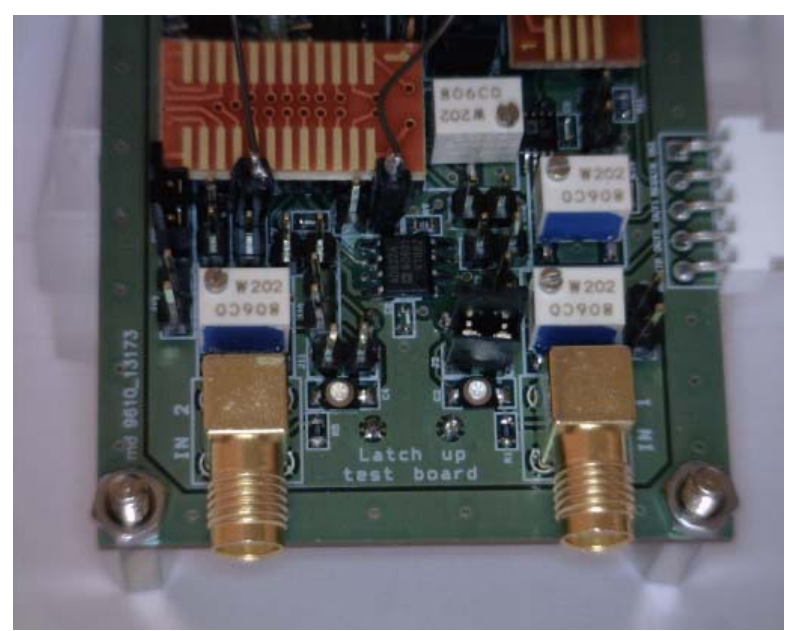

Figure 3: The board

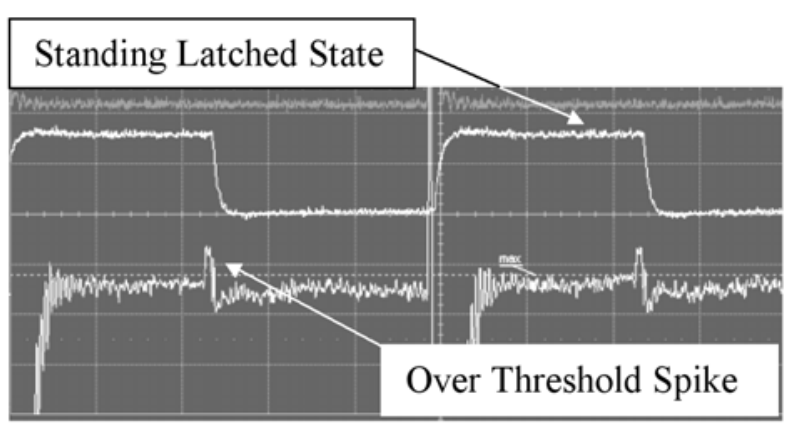

Figure 4: Oscilloscope plot at $\mathrm{T}=5 \mu \mathrm{s}$ 
was no longer necessary to raise again the height of the pulse. The same approach was used by decreasing the height of the pulse starting from the latter situation till the circuit did not ignite at any time because the height was too low. These measurements have been repeated several times to estimate the reliability and repeatability of the system. Figure 5 shows a test configuration using a cyclic ignition of the system. Figure 6

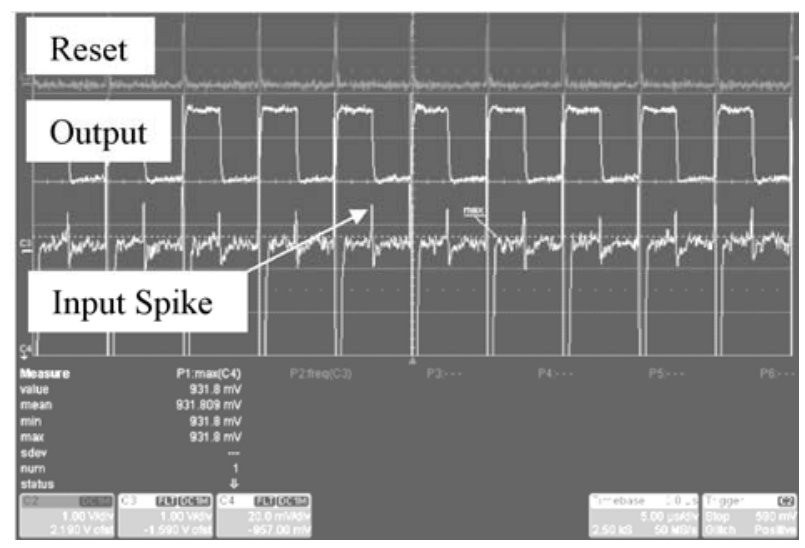

Figure 5: Cyclic latchup ignitions at $\mathrm{T}=5 \mu \mathrm{s}$

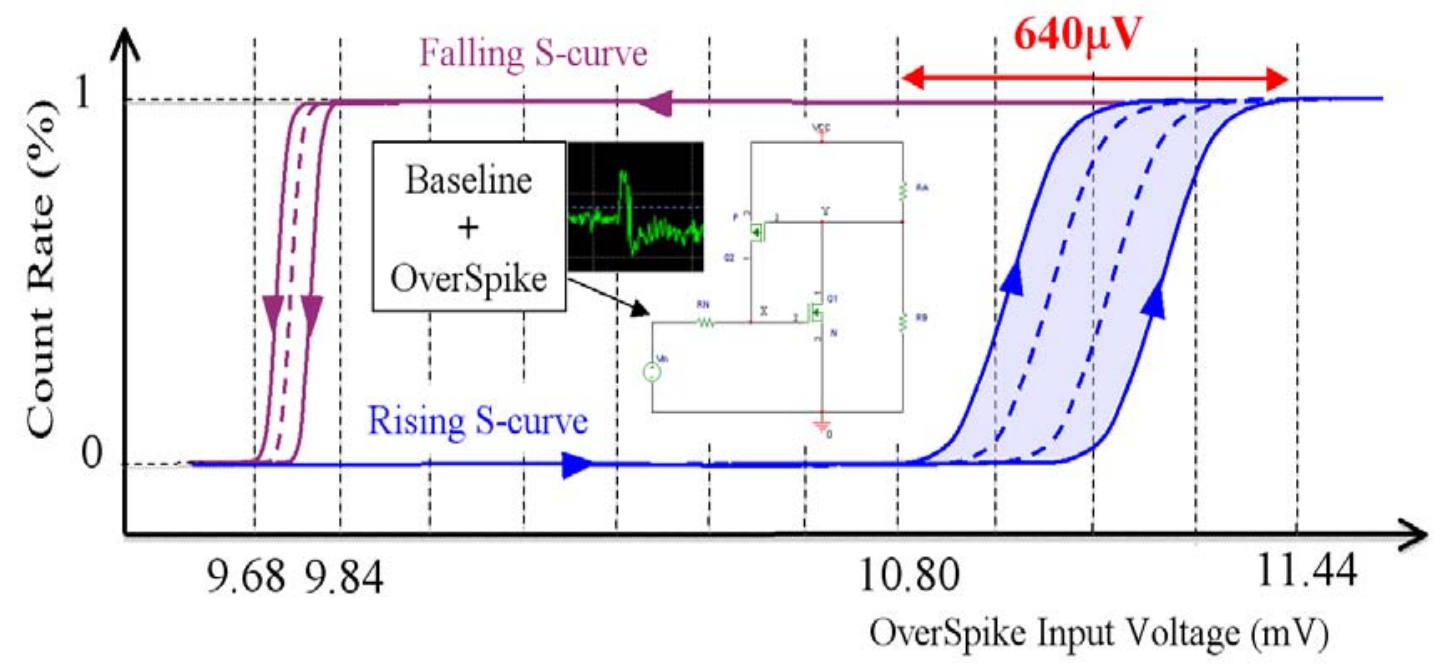

Figure 6: Noise curve

summarizes the measurements pointing out that, repeating the tests, the non-ignition to ignition transition points varied. Additionally, this variation occurred in either ways, by increasing and by decreasing the pulse height. This can be interpreted as the uncontrolled noise that affected the measurements. It can be easily seen that the rising and falling curves of the noise transition S-curves - are different in transition width, point and spread. However, the most significant part of the curve has been shaded to point out that the spread in the ignition point is about $640 \mathrm{mV}$ i.e. spread of ignition threshold @ 50\% of S-curve, let us say lower that $1 \mathrm{mV}$, in any case -. So, both rising and falling transition spreads are very sharp since any transition curve owns a noise that can be estimated in of the order of $100 \mathrm{mV}$. This seems to be very promising for future applications in particle detectors or signal readout systems, being the threshold already very precise (low noise) using commercial components. All in all, the whole power consumption of the cell is also very low, of the order of $1 \mathrm{~mW}$, when it is not ignited. This can be easily understood since the number of components inserted, basically two transistors plus one reset switch plus some resistors, is much smaller than that of the modern pixel circuits. Hence, it is reasonable to expect even better numbers and results for integrated versions of the latchup circuit. The authors [see G. Villani et al., 8, 9] are investigating other types of latchup detector 
studies oriented to low-power applications and dosimetry. In fact, if just one or both transistors of the latchup cell are replaced with floating gate devices, not only the over spike input would be under control, but also the baseline over which this spike is added. Thus, being the charge injected within the floating gate removable via external radiation, the same latchup circuit could be applied as a dosimeter. In more detail, once a floating gate MOS has been programmed with a certain threshold, this threshold is swept back down depending on the total absorbed radiation dose, till the latchup process ignites spontaneously. Hence, if the threshold to absorbed dose ratio is known, it can be claimed that the cell ignites whenever a certain dose of radiation is absorbed: this is a dosimeter. This type of research is ongoing but the principle has already been proved $[8,9]$.

\section{The MESFET}

This section describes the simulation and the realization of a thyristor topology using one Pchannel MOS transistor and one N-channel 24010 MESFET SiC by CREE [10]. The ignition is confirmed as it was investigated in the past provided a different polarization. Figure 7 shows essentially the circuit mounted on a test-board, along with the values of the components. Figure 8 shows two hard-plots of the circuit's behavior. The green lines represent the Vout in the circuit while the pink plots are the MESFET's gate, or Vin pin. The difference on the pink plots depends on the scale. Although the input spike is not visible, it is clear that the two curves cross each other as a confirmation of the ignition. The voltage swing is of the order of $2 \mathrm{~V}$ and the ignition time of the order of several milliseconds. All in all we plan to investigate in depth the circuit by fabricating a better and dedicated test-board to allow sensitivity estimation measurements.

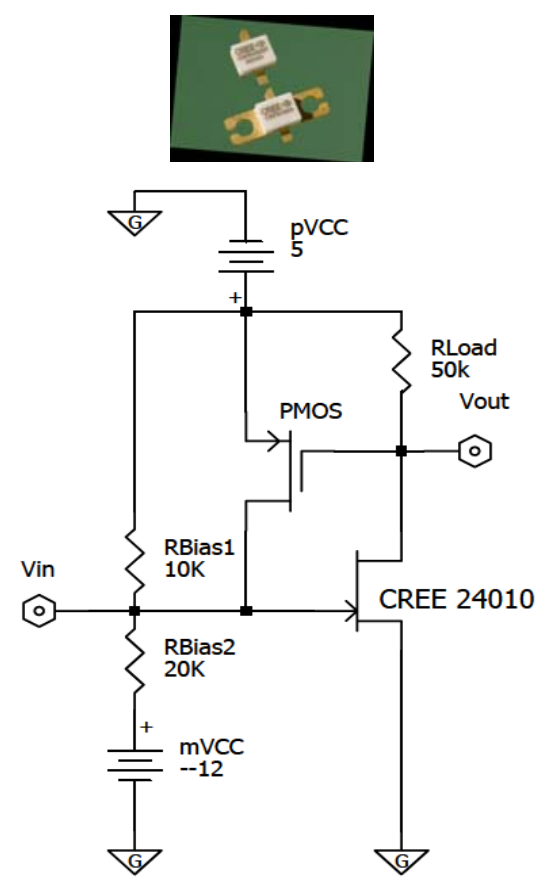

Figure 7: Actual circuit mounted on a test-board using the MESFET 24010

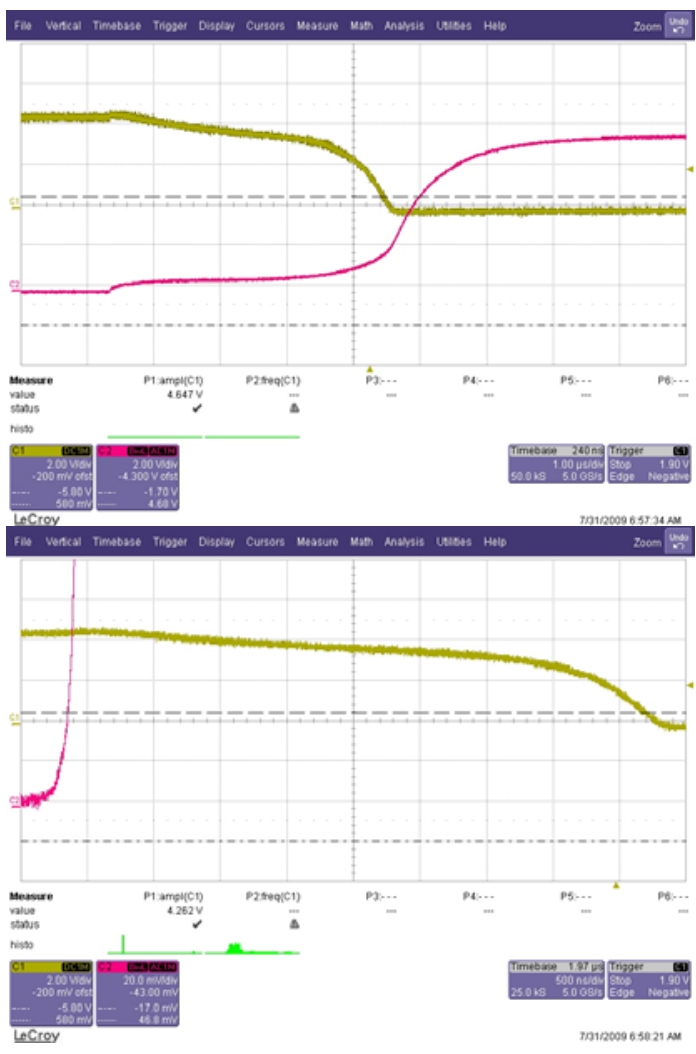

Figure 8: Oscilloscope plots for the circuit with MESFET 24010 


\section{Conclusion}

This study indicates that a very simple circuit can operate like the more complicated structures used today in modern pixel detectors. In principle, an integrated device designed via modern CMOS technologies may work as particle detector, as readout circuit for general sensors provided the increased performance. The cell tested in laboratory was designed by exploiting MOS transistors implemented in commercial CMOS inverters and these transistors were connected to form a thyristor device. The circuit has a noise spread of the threshold lower that $1 \mathrm{mV}$, power consumption due to leakage currents of the order of $1 \mathrm{~mW}$, estimated charge sensitivity of the order of $1 \mathrm{pC}$ and very good repeatability.

For the MESFET component we have just mounted a very rough circuit without paying attention to the parasitic effects and we have not investigated the sensitivity of the circuit. This will be a future work. For the time being the results confirm that also a MESFET component can be used into a latchup topology for detection application in high-energy physics.

Possible applications range from heavy-ion discriminator to beam monitors provided they deposit an over-threshold charge in the cell. In fact, after the latchup ignition, the cell retains the position of the crossing particle by means of a self-locked thyristor that provides noiseless, digital, robust and stable signal until the whole system has been powered off.

\section{References}

[1] F.W. Sexton, "Destructive single-event effects in semiconductor devices and ICS', IEEE Trans. Nucl. Sci., 2003, 50/3, 603-621

[2] A.H. Johnston, "Latchup in integrated circuits from energetic protons", IEEE Trans. Nucl. Sci., 1997, 44/6, 2367-2377

[3] G. Gagliardi, “The ATLAS pixel detector electronics” Nucl.Instr. Meth. A”, 2001, 466 275-281

[4] R. Baur, "Readout architecture of the CMS pixel detector", Nucl. Instr. Meth. A, 2001, 465, 159165

[5] P. Riedler et al., "Production and integration of the ALICE silicon pixel detector", Nucl. Instr. Meth. A 2006, 572, 128-131

[6] A. Gabrielli, "Proposal for solid-state particle detector based on latchup effect”, El. Lett., 2005, 41/11, 641-643

[7] A. Gabrielli, "Particle detector prototype based on a discrete-cell sensitive to latchup effect", Meas. Sci. Tech., 2006, 17, 2269-2273

[8] G. Villani, A. Gabrielli, D. Demarchi, "A family of sensitive pixel devices by exploiting the latchup effect”, SORMA WEST 2008, Proceedings of the Symposium on Radiation Measurements and Applications 02-05 June, 2008, Berkeley, CA, USA

[9] G. Villani et al., "Radiation detection and readout based on the latchup effect" - Proceedings of the PSD 2008 - Int. Conf. on Position Sensitive Detectors, 01-05 September, 2008, Glasgow, UK, to be published in Nucl. Instr. Meth. A

[10] http://www.cree.com 